\title{
Interactive Modification of Real and Virtual Lights for Augmented Reality
}

\author{
Céline Loscos, George Drettakis and Luc Robert
}

iMAGIS*- GRAVIR/IMAG - INRIA

\section{Introduction and Previous Work}

Computer augmented reality (CAR) provides the ability to mix real and virtual worlds, and is used in entertainment, television, or help systems for repair and manufacturing. Reconstructed real world models based on video images obviate the need for the often arduous task of modeling complex scenes in detail. We focus here on common illumination, that is the interaction of shadows and other lighting effects between real and virtual objects. We build on the approach of [DRB97], for which vision techniques are used to extract an approximate model of the scene. Based on work by Fournier et al. [FGR93], a basic representation of illumination is built using a radiosity algorithm. Virtual objects can be inserted, using the incremental hierarchical radiosity (HR) approach of [DS97].

In this abstract we present novel solutions which allow the addition of virtual lights and the modification of real light source intensities. The main problem is that real world lights cast shadows which are embedded in the textures of the model used to reconstruct the real world. No previous solutions exist to the problem of identification and removal of these shadows, thus permitting light modification.

Our solution is based on a pre-processing step which modifies the textures by weighting them according to shadow relationships identified in the radiosity solution. A correction is then applied re-instating an approximation of the original shadows. Real lights can then be turned off or their intensities changed interactively, and virtual lights and objects can also be rapidly inserted.

\section{Initialization of the Radiosity System}

In [FGR93] and [DRB97] radiosity parameters are retrieved from the textures extracted from the real scene images. In particular, real light source emittance and object reflectance is estimated based on texture values and simple assumptions on average scene reflectance and radiosity. After this estimation, the geometry representing the real scene is subdivided into patches with radiosity $B_{i}^{o}$ (original radiosity). After adding a virtual object, modified radiosities (i.e. in the shadow of a virtual object), are $B_{i}$. The texture $t_{i}$ of each patch is modulated by the ratio $\frac{B_{i}}{B_{i}^{o}}$ (Eq. (1)).

The ratio of Eq. (1) is insufficient to remove shadows when we change the real light intensities, since the texture $t_{i}$ already contains the shadow due to the real sources. To perform this correctly, we need to remove the shadow from the texture and represent it explicitly using the radiosity solution.

For each patch in shadow (as determined by the radiosity system), we compute a value $\bar{E}_{i}$, which is the irradiance which would have arrived at patch $i$, but was blocked by occlusion. To generate a modified texture, we compute a correction factor based on $\bar{E}_{i}$ for patches in shadow. Unoccluded patches are displayed using the ratio of Eq. (1) which has unit value, since no virtual object has yet been inserted. The resulting image will be used to replace the original texture.

For patch $i$ and $s=1 . . N$ sources with radiosities $B_{S}$, we use the following correction factor: $\frac{\rho_{j} \sum_{s=1 . . N}\left(F_{s i} B_{s}+\bar{E}_{i}\right)}{B_{i}^{o}} \times t_{i}$, where $B_{i}^{o}$ is the

*iMAGIS is a joint research project of CNRS/INRIA/UJF/INPG. iMAGIS/GRAVIR, BP 53, F-38041 Grenoble Cedex 09 France. E-mail: \{Celine.Loscos|George.Drettakis\}@imag.fr original radiosity value and $F_{s i}$ the form-factor from $s$ to patch $i$. We scale by the reflectance $\rho_{j}$ of a user-selected patch, which is not in shadow and has the appropriate color i.e. of the shadowed surface.

For each patch corresponding to the modified texture, we update the reflectance as before and set the original radiosity to $B_{i}^{o}=\rho_{i}\left(E_{i}+\bar{E}_{i}\right)$, capturing the radiosity changes from the texture. The radiosity is then recomputed by solving the HR system. To compensate for approximation errors, a display correction factor is used based on previous texture values for modified patches.

\section{Interactive modification of Real Lights}

We can now interactively modify the illumination of the real light sources. While maintaining links and subdivision, we first remove the radiosity and iteratively gather and push-pull, thus updating radiosity based on the new emittance value of a source. For example, if we switch off a light, the corresponding shadows are no longer perceptible in the image. We can also insert a virtual light source in the hierarchy, and project the shadows of the real scene objects. Figure 4 shows the initial illumination on the left, and on the right, the same real scene with all of the four real light sources switched off and a virtual light source switched on.

\section{Conclusion and Future Work}

We have developed a solution to remove real objects shadows and to perform interactive modifications of both real and virtual illumination. Shadows could be more accurately delimited during texture modification using a color-based refinement.

Our method could be extended to remove real objects and insert virtual replacements, since shadow removal is the hardest problem in this case. Since the reconstruction of the real world model is never perfect, we expect to increase user interaction to dynamically determine the level of geometric detail required during an interactive session.
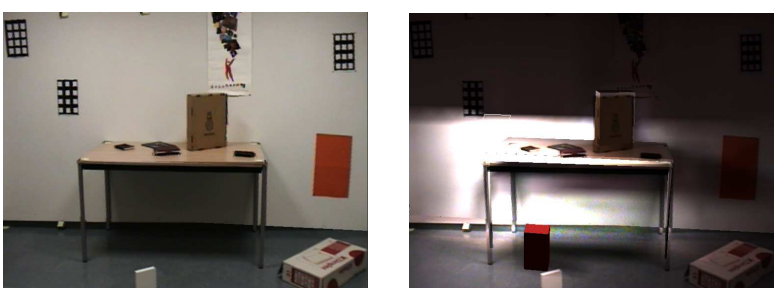

Figure 1: (a) Initial illumination, (b) Modified illumination

\section{References}

[DRB97] G. Drettakis, L. Robert, and S. Bougnoux. Interactive common illumination for computer augmented reality. In J. Dorsey and P. Slusallek, eds., Rendering Techniques '97, pages 45-56, 8th EG workshop on Rendering, Saint Etienne, 1997. Springer Verlag.

[DS97] G. Drettakis and F. X. Sillion. Interactive update of global illumination using A line-space hierarchy. In T. Whitted, ed., SIGGRAPH 97 Conference Proceedings, Annual Conference Series, pp 57-64, 1997.

[FGR93] A. Fournier, A. S. Gunawan, and C. Romanzin. Common illumination between real and computer generated scenes. In Proc. of Graphics Interface '93, pp 254-262, Toronto, Ontario, Canada, May 1993. CIPS. 